\title{
Polymorphisms in TWIST1 and ZEB1 Are Associated with Prognosis of Gastric Cancer Patients
}

\author{
SANDRA ROMERO $^{1}$, MAHER MUSLEH $^{2,3}$, MARCO BUSTAMANTE $^{4}$, JUAN STAMBUK ${ }^{5}$, \\ RAUL PISANO ${ }^{6}$, ENRIQUE LANZARINI ${ }^{2,3}$, HECTOR CHIONG $^{7}$, JORGE ROJAS $^{3}$, V GONZALO CASTRO $^{1}$, \\ LILIAN JARA $^{1}$, ZOLTAN BERGER $^{2}$ and PATRICIO GONZALEZ-HORMAZABAL ${ }^{1}$ \\ ${ }^{1}$ Human Genetics Program, Institute of Biomedical Sciences (ICBM), \\ School of Medicine, University of Chile, Santiago, Chile; \\ ${ }^{2}$ Department of Gastroenterology, University of Chile Clinical Hospital, Santiago, Chile; \\ ${ }^{3}$ Department of Surgery, University of Chile Clinical Hospital, Santiago, Chile; \\ ${ }^{4}$ Department of Surgery, School of Medicine at Eastern Campus, University of Chile, Santiago, Chile; \\ ${ }^{5}$ Department of Surgery, San Juan de Dios Hospital, Santiago, Chile; \\ ${ }^{6}$ Department of Pathology, San Juan de Dios Hospital, Santiago, Chile; \\ ${ }^{7}$ Department of Surgery, Barros Luco Hospital, Santiago, Chile
}

\begin{abstract}
Background/Aim: Epithelial-mesenchymal transition (EMT) program has been linked as a driver of metastatic dissemination by conferring migratory and invasive capacity to cancer cells. Gastric cancer (GC) patients with tumors expressing altered levels of EMT markers have low survival. This study aimed to assess if polymorphisms of CDH1, TWIST1, SNAIL2, ZEB1 and ZEB2 genes are associated with survival in GC patients. Patients and Methods: A total of 153 individuals with diagnosis of GC were recruited in Santiago, Chile. All patients were genotyped using Infinium Global Screening Array (GSA). Twenty Tag SNPs of the studied genes were retrieved. Results: Three SNPs were associated with survival: rs2526614 (TWIST1) (genotype CA + AA, adjusted $H R=0.58$, 95\%CI $=0.37$ 0.93), rs6953766 (TWIST1) (genotype $G G$, crude $H R=2.02$, 95\%CI=1.06-3.82, adjusted $H R=2.14,95 \% C I=1.07-4.25)$, and rs431073 (ZEB1) (genotype $A C+C C$, crude $H R=1.62$, 95\%CI=1.01-2.59, adjusted $H R=1.96,95 \% C I=1.18-3.25$ ). Conclusion: To the best of our knowledge, this is the first study proposing a role of these SNPs in cancer prognosis. Their use as prognostic markers of GC survival warrants further investigation.
\end{abstract}

This article is freely accessible online.

Correspondence to: Patricio Gonzalez-Hormazabal, Human Genetics Program, Institute of Biomedical Sciences (ICBM), School of Medicine, University of Chile, Av. Independencia 1027, Santiago, Chile. Tel: +56 229786845, e-mail: patriciogonzalez@ uchile.cl.

Key Words: Gastric cancer, polymorphisms, SNP, TWIST, ZEB, epithelial-to-mesenchymal transition.
Gastric cancer (GC) is the sixth most common cancer and the third leading cause of death due to cancer worldwide (1). Mortality due to GC varies according to world region, from 15.2 age-standardized rate (ASR) per 100,000 in East Asia to 2.3 ASR per 100,000 in North America. Chile has a high GC mortality, with 16.7 ASR per 100,000 predicted for 2017; and has one of the highest GC mortality rates worldwide $(1,2)$.

More than half of patients are diagnosed at an advanced stage (TNM III-IV), where the 5-year overall survival (OS) rate is less than $50 \%$ (3). In Chile, it was estimated that 3 out of 4 cases are diagnosed with stage III or IV, with a 5-year survival of $10.6 \%$ (4). The stage of cancer at diagnosis is a key factor in defining prognosis and therefore it is a critical element in determining appropriate treatment. The most recognized evidence-based GC staging system in practice is the tumor-node-metastasis (TNM) concept from the American Joint Committee on Cancer (AJCC) (5). The AUC (area under the curve) of TNM staging to predict GC survival is near to 0.8 (6). Since this value is below 1 (considered "perfect prediction"), it suggests that other factors such as host characteristics play a role in GC prognosis. A handful of single nucleotide polymorphisms (SNPs) have been described as risk factors for GC (7), and there is growing evidence associating SNPs with GC survival (8).

Metastasis is estimated to be responsible for over $90 \%$ of patient mortality associated with solid tumors (9). Epithelial-mesenchymal transition (EMT) is known as a driver of metastatic dissemination by conferring migratory and invasive capacity to cancer cells, allowing them to get a cancer stem cell state (10). EMT is a program which transforms epithelial cells into mesenchymal ones. This program is involved not only in cancer, but also in embryonic 
development, wound healing and fibrosis (11). EMT is triggered by various signals including fibroblast growth factors (FGFs) and the Wnt pathway (10). Canonical downstream effectors include EMT transcription factors belonging to zinc finger or bHLH families, such as Snail, Zeb, and Twist. These factors suppress the expression of the prototypical adhesion protein E-cadherin at the transcriptional level (11). Interestingly, mutations in $\mathrm{CDHI}$ (the gene encoding Ecadherin) are responsible for hereditary diffuse gastric cancer. EMT is closely related to GC and levels of EMT markers are all up-regulated in patients with dysplasia or early GC, while the level of E-cadherin is decreased in these patients (12). GC patients with tumors expressing low levels of E-cadherin and high levels of Snail, Zeb and Twist have low survival (13-15).

Thus far, there are no studies that investigate the prognostic value of polymorphisms in EMT genes in GC patients. In the present study, it was examined whether variants of $C D H 1$, TWIST1, SNAIL2, ZEB1 and ZEB2 genes are associated with $\mathrm{OS}$ in GC patients.

\section{Patients and Methods}

Patients. A total of 153 individuals with a preoperative diagnosis of gastric adenocarcinoma were recruited at the time of surgical resection between December 2010 and August 2014 from different hospitals located in Santiago de Chile: University of Chile Clinical Hospital, Salvador Hospital, Barros Luco Trudeau Hospital, and San Juan de Dios Hospital. Only cases with a postoperative histopathological diagnosis of gastric adenocarcinoma were included. In all cases, the tumor was located distally from the cardia. Passive follow-up was performed obtaining death report from Civil Registry and Identification Service of Chile. The last follow up was in January 2018. OS was considered as the endpoint. Patients alive on the last follow-up were considered censored. Tumor size, depth of invasion, and lymph node metastasis were obtained from the histopathological report. Lauren's criteria were used to classify tumors as intestinal or diffuse.

Ethical approval. This study was approved by the Ethical Committee of the following institutions: University of Chile School of Medicine (\#023/2011), University of Chile Clinical Hospital (\#029/2011), Metropolitan South-Santiago Public Health Agency (\#MK523B-118), Metropolitan East-Santiago Public Health Agency (\#24/01/2012), and Metropolitan West-Santiago Public Health Agency (\#236/2009). All participants gave their written informed consent. The study was performed in accordance with the Declaration of Helsinki.

Genotyping and SNP selection. Genomic DNA was isolated from peripheral blood leukocytes using salting out method and Proteinase $\mathrm{K}$ or according to the method described by Chomczynski and Sacchi (16). In both cases, genomic DNA was repurified using Monarch PCR and DNA Cleanup columns (NEB, MA, USA). DNA samples were genotyped using Infinium Global Screening Array (Illumina, CA, USA) according to manufacturer's instructions in the Human Genotyping Facility (HuGe-F) at Erasmus MC, The Netherlands. Per-individual and Per-marker quality controls were performed according to the guideline of Anderson et al. (17). All the studied patients and SNPs passed the quality control otherwise indicated. Studied genes were $C D H 1$, TWIST1, SNAIL, ZEB1, and ZEB2. The analyzed SNPs were selected from the manifest file of the GSA array according to the following criteria: (1) located between $5 \mathrm{~kb}$ upstream of transcription start site and $5 \mathrm{~kb}$ downstream of the stop triplet according to GRCh37 assembly of human genome; (2) minor allele frequency (MAF) higher than 0.16; (3) not in linkage disequilibrium (LD) $(\mathrm{r} 2>0.8)$ with other SNPs contained in the array; and (4) with no departure from Hardy-Weinberg equilibrium (HWE) in the studied population $(p<0.05)$. The final list of the analyzed SNPs was: $C D H 1$ : rs16260, rs2098728, rs7186053; TWIST1: rs2526614, rs10240058, rs10228406, rs6953766, rs17140672, rs73325513; SNAIL2: rs2582778; ZEB1: rs431073; ZEB2: rs35339313, rs16823675, rs13013418, rs7597006, rs7599224, rs12327962, rs13382811, rs12691693, rs6740731.

Statistical analyses. A forward stepwise Cox regression model was used as variable selection procedure to obtain a candidate model to predict GC survival including SNPs (additive model) and clinicopathological variables, using $p<0.05$ for addition from the model and $p>0.051$ for removal from the model. The assumption of proportional hazards was tested according to Gramsch and Therneau (18) for all the selected variables. Hazard Ratios (HR) were estimated from Cox regression models using the selected variables as predictors. The association of polymorphisms with survival was also assessed with the log-rank test and Kaplan-Meier method. Median Survival Time (MST) was estimated at the 50th percentile, otherwise indicated. The Hausman specification test (19) was used to assess if there are differences between beta coefficients from two Cox regression models. $p$-Values were 2 -sided and $p<0.05$ was considered statistically significant. All statistical analyses were performed using Stata 12 (StataCorp LLC, TX, USA). For SNPs, allele (additive), dominant and recessive models were considered.

\section{Results}

Patient characteristics. The demographic characteristics and clinicopathological features of the 153 patients included in the study are shown in Table I. The age at diagnosis ranges between 29 and 88 years, mean of 65.9 years, median 68 years and standard deviation of 10.9 years. Median follow-up time was 56.4 months $(95 \% \mathrm{CI}=54.2-59.7)$ according to reverse $\mathrm{K}-$ $\mathrm{M}$ method (20). The MST of the 153 included patients was 42 months $(95 \% \mathrm{CI}=27.2-67.1)$. Of the 153 patients, $85(55.6 \%)$ died during the follow-up period. The advanced stage was more prevalent at diagnosis than early stage: $49.7 \%$ had tumors with $\mathrm{T} 4$ invasion, and $72.5 \%$ were positive for lymph node (N1, N2 or N3). In the univariate analysis (Table I), tumor size $>5 \mathrm{~cm}$, Lauren's diffuse-type (or mixed), depth of invasion T3 and T4, and Lymph node metastases N1, N2 or $\mathrm{N} 3$ were significantly associated with poor OS.

SNPs proposed as independent predictors of overall survival. A forward stepwise Cox regression analysis (Table II) with all the 20 SNPs was performed using the additive model, and age, gender, tumor size, histological type, depth of invasion and lymph node metastases as potential prognostic factors. 
Table I. Association of demographic characteristics and clinicopathological features with overall survival.

\begin{tabular}{|c|c|c|c|c|c|c|}
\hline & Patients & Deaths & MST (months) (a) & Log-rank $p$-Value & HR (95\%CI) (b) & $\operatorname{HR}(95 \% \mathrm{CI})(\mathrm{c})$ \\
\hline \multicolumn{7}{|l|}{ Age } \\
\hline$<=60$ years & 46 & $21(45.7 \%)$ & 17.9 & 0.11 & 1 & \\
\hline$>60$ years & 107 & $64(59.8 \%)$ & 9.3 & & $1.49(0.91-2.44)$ & \\
\hline \multicolumn{7}{|l|}{ Gender } \\
\hline Female & 49 & $24(49.0 \%)$ & 9.8 & 0.41 & 1 & \\
\hline Male & 104 & $61(58.7 \%)$ & 12 & & $1.22(0.76-1.96)$ & \\
\hline \multicolumn{7}{|l|}{ Tumor size } \\
\hline$<=5 \mathrm{~cm}$ & 53 & $21(39.6 \%)$ & 37.7 & $<0.01$ & 1 & \\
\hline$>5 \mathrm{~cm}$ & 100 & $64(64.0 \%)$ & 9.3 & & $2.19(1.34-3.60)$ & $2.22(1.34-3.67)$ \\
\hline \multicolumn{7}{|l|}{ Histological type } \\
\hline intestinal & 100 & $49(49.0 \%)$ & 17.8 & 0.01 & 1 & \\
\hline Diffuse/mixed & 53 & $36(67.9 \%)$ & 8.6 & & $1.74(1.13-2.68)$ & $1.93(1.24-3.00)$ \\
\hline \multicolumn{7}{|l|}{ Depth of invasion } \\
\hline $\mathrm{T} 1$ & 20 & $4(20.0 \%)$ & 67.1 & $<0.01$ & 1 & \\
\hline $\mathrm{T} 2$ & 13 & $6(46.2 \%)$ & 35.1 & & $2.74(0.779 .71)$ & $2.70(0.73-1.88)$ \\
\hline $\mathrm{T} 3$ & 44 & $23(52.3 \%)$ & 12 & & $3.71(1.28-10.8)$ & $3.63(1.36-10.5)$ \\
\hline $\mathrm{T} 4$ & 76 & $52(68.4 \%)$ & 8.6 & & $5.46(1.97-15.1)$ & $3.28(2.00-15.3)$ \\
\hline \multicolumn{7}{|c|}{ Lymph node metastasis } \\
\hline No & 42 & $9(21.4 \%)$ & 67.1 & $<0.001$ & 1 & \\
\hline $\mathrm{N} 1 / \mathrm{N} 2 / \mathrm{N} 3$ & 111 & $76(68.5 \%)$ & 15 & & $4.96(2.48-9.92)$ & $4.76(2.37-9.58)$ \\
\hline Total & 153 & $85(55.6 \%)$ & 24.3 & & & \\
\hline
\end{tabular}

(a) Median Survival Time at percentile 25th; (b) Hazard Ratio (95\% Confidence Interval), crude; (c) Hazard Ratio (95\% Confidence Interval), adjusted for age and gender.

Table II. Variables included in the model after a stepwise Cox regression analysis.

\begin{tabular}{lccccc}
\hline Variable & category & beta & SE & $p$-Value & HR (95\%CI) \\
\hline Lymph node metastasis & N1, N2, N3 & 1.76 & 2.08 & 0.001 & $5.79(2.86-11.71)$ \\
rs2526614 & allele A & -0.36 & 0.11 & 0.03 & $0.70(0.51-0.96)$ \\
rs6953766 & allele G & 0.46 & 0.26 & $<0.01$ & $1.59(1.15-2.20)$ \\
rs431073 & allele C & 0.37 & 0.23 & 0.02 & $1.45(1.07-1.97)$ \\
\hline
\end{tabular}

beta: Coefficient of Cox regression; SE: standard error. HR (95\%CI) Hazard Ratio (95\% Confidence Interval).

rs2526614 (TWIST1), rs6953766 (TWIST1) and rs431073 (ZEB1), as well as lymph node metastases stage $\mathrm{N} 1, \mathrm{~N} 2$ or N3 were identified as independent predictors of OS in GC patients.

Association of rs2526614 (TWIST1), rs6953766 (TWIST1) and rs431073 (ZEB1) with gastric cancer overall survival. HR for rs2526614 (TWIST1) was only significant under the dominant model (CA+AA) adjusted by clinicopathological variables (Table III). Nevertheless, there were no significant differences between hazard function of CA+AA versus CC subjects (Logrank $p$-value $=0.24$, Figure 1). Genotype GG of rs6953766 (TWIST1) was significantly associated with poor OS, both crude $(\mathrm{HR}=2.02,95 \% \mathrm{CI}=1.06-3.82)$ and adjusted by clinicopathological variables $(\mathrm{HR}=2.14,95 \% \mathrm{CI}=1.07-4.25)$
(Table III). Comparing Kaplan-Meier estimator curves, GG genotype has the worst survival time (MST=9.8 months) compared with TT or TG genotypes (MST=46 months) (Figure 1), nevertheless the difference did not reach statistical significance (Log-rank $p$-value=0.18). Carriers of the $\mathrm{C}$ allele of rs431073 (ZEB1) have a worse prognosis compared to those with the AA genotype. Adjusted Cox regression analyses showed that this polymorphism is associated with OS under the dominant model (crude $\mathrm{HR}=1.62,95 \% \mathrm{CI}=1.01-2.59$, adjusted $\mathrm{HR}=1.96,95 \% \mathrm{CI}=1.18-3.25$ ) (Table III). According to KaplanMeier estimator curves, carriers of rs431073 C allele (CC or CA genotypes) have a low OS (MST=17.9 months) compared to patients with the AA genotype (MST=38.5 months) (Logrank $p$-value 0.04, Figure 1). 
A

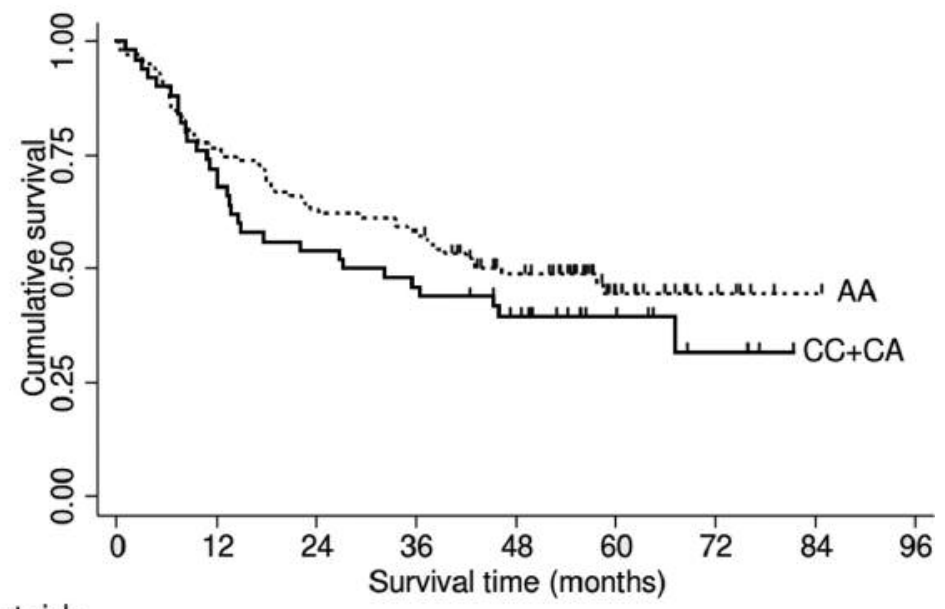

Number at risk

$\begin{array}{rccccccccc}\text { rs } 2526614=\mathrm{CC}+\mathrm{CA} & 50 & 36 & 27 & 23 & 17 & 8 & 3 & 0 & 0 \\ \text { rs2526614 = AA } 103 & 79 & 65 & 60 & 42 & 16 & 6 & 1 & 0\end{array}$

B

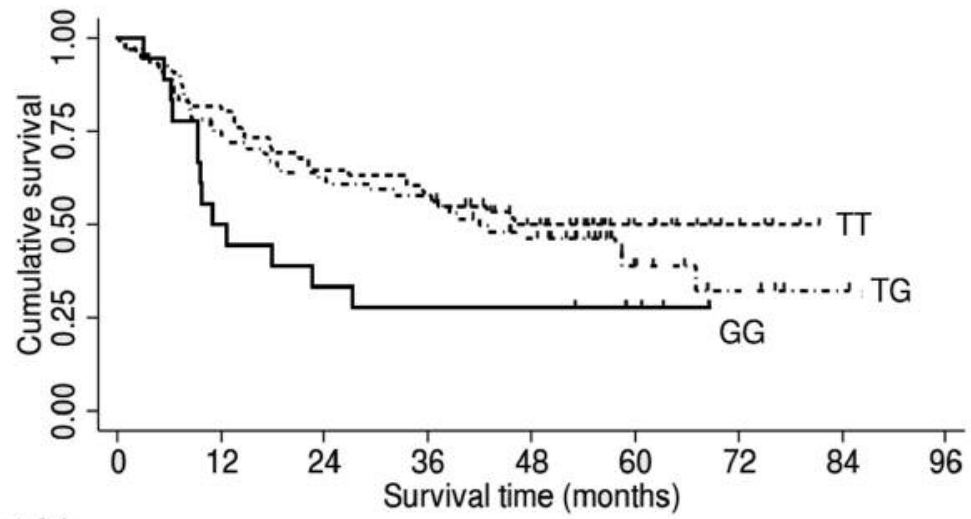

\begin{tabular}{|c|c|c|c|c|c|c|c|c|c|}
\hline \multirow{2}{*}{$\begin{array}{r}\text { Number at risk } \\
\text { rs6953766 = TT }\end{array}$} & \\
\hline & 71 & 58 & 46 & 41 & 28 & 12 & 5 & 0 & \\
\hline rs $6953766=\mathrm{TG}$ & 64 & 48 & 40 & 37 & 26 & 9 & 4 & 1 & \\
\hline rs6953766 = GG & 18 & 9 & 6 & 5 & 5 & 3 & 0 & 0 & \\
\hline
\end{tabular}

C

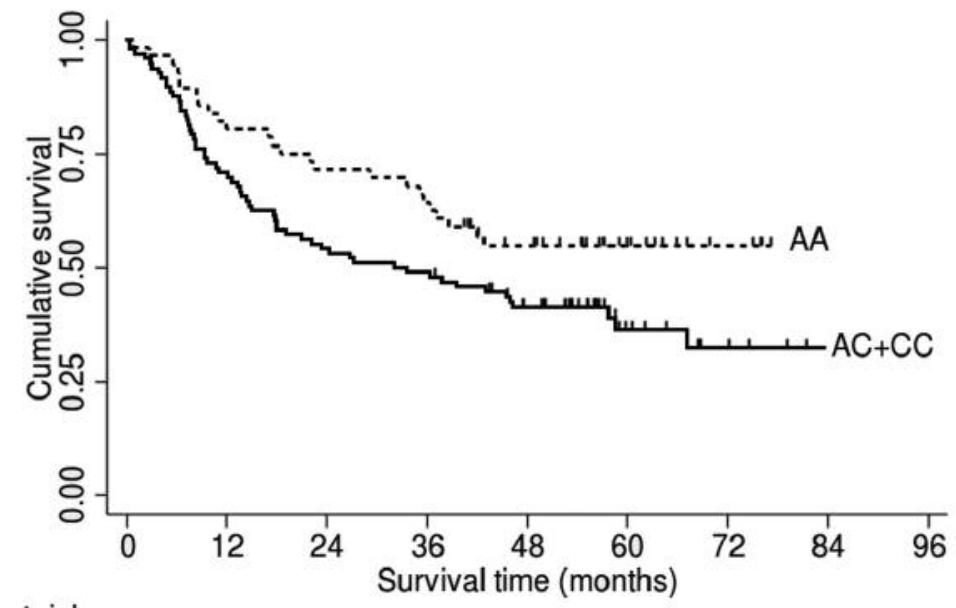

Number at risk

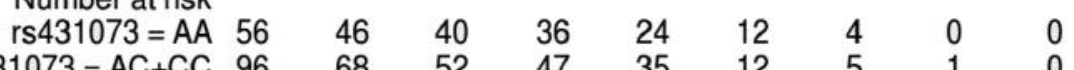

Figure 1. Kaplan-Meier curves of overall survival for (A) rs2526614, (B) rs6953766, (C) rs431073, in gastric cancer patients. 
Table III. Cox regression analyses for association of rs2526614 (TWIST1), rs6953766 (TWIST1) and rs431073 (ZEB1) with gastric cancer overall survival.

\begin{tabular}{|c|c|c|c|c|c|c|c|c|}
\hline & Patients & $\begin{array}{l}\text { Deaths } \\
(\%)\end{array}$ & $\begin{array}{l}\text { MST (months) } \\
\text { (a) }\end{array}$ & $\begin{array}{c}\text { Log-rank } \\
p\end{array}$ & $\begin{array}{c}\text { Crude HR } \\
(95 \% \mathrm{CI})\end{array}$ & $p$-Value & $\begin{array}{l}\text { Adjusted HR } \\
(95 \% \mathrm{CI})(\mathrm{b})\end{array}$ & $p$-Value \\
\hline \multicolumn{9}{|c|}{ rs2526614 (TWIST1) } \\
\hline $\mathrm{CC}$ & 50 & $31(62.0 \%)$ & 14.7 & \multirow[t]{3}{*}{$0.47(\mathrm{c})$} & 1.00 & 1.00 & 1.00 & \\
\hline $\mathrm{CA}$ & 72 & $38(52.8 \%)$ & 29.2 & & $0.79(0.49-1.27)$ & 0.33 & $0.56(0.34-0.92)$ & 0.02 \\
\hline AA & 31 & $16(51.6 \%)$ & 42.0 & & $0.72(0.39-1.31)$ & 0.28 & $0.66(0.35-1.24)$ & 0.20 \\
\hline $\mathrm{CC}$ & 50 & $31(62.0 \%)$ & 14.7 & \multirow[t]{2}{*}{$0.24(\mathrm{~d})$} & 1.00 & 1.00 & 1.00 & \\
\hline $\mathrm{CA}+\mathrm{AA}$ & 104 & $55(52.9 \%)$ & 33.6 & & $0.77(0.49-1.19)$ & 0.24 & $0.58(0.37-0.93)$ & 0.02 \\
\hline \multicolumn{9}{|c|}{ rs6953766 (TWIST1) } \\
\hline TT & 71 & $35(49.3 \%)$ & 35.1 & \multirow[t]{2}{*}{0.09} & 1.00 & 1.00 & 1.00 & \\
\hline TG & 64 & $37(57.8 \%)$ & 29.2 & & $1.20(0.76-1.91)$ & 0.43 & $1.44(0.89-2.31)$ & 0.14 \\
\hline GG & 18 & $13(72.2 \%)$ & 9.8 & \multirow{3}{*}{0.18} & $2.02(1.06-3.82)$ & 0.03 & $2.14(1.07-4.25)$ & 0.03 \\
\hline TT & 71 & $35(49.3 \%)$ & 35.1 & & 1.00 & 1.00 & 1.00 & \\
\hline $\mathrm{TG}+\mathrm{GG}$ & 82 & $50(61.0 \%)$ & 18.5 & & $1.34(0.87-2.07)$ & 0.18 & $1.56(0.99-2.44)$ & 0.05 \\
\hline \multicolumn{9}{|c|}{ rs431073 (ZEB1) } \\
\hline AA & 56 & $25(44.6 \%)$ & 38.5 & \multirow[t]{3}{*}{0.12} & 1.00 & 1.00 & 1.00 & \\
\hline $\mathrm{AC}$ & 70 & $43(61.4 \%)$ & 17.7 & & $1.63(0.99-2.67)$ & 0.53 & $1.91(1.13-3.22)$ & 0.02 \\
\hline $\mathrm{CC}$ & 26 & $16(61.5 \%)$ & 19.0 & & $1.60(0.85-3.00)$ & 0.14 & $2.14(1.10-4.19)$ & 0.03 \\
\hline AA & 56 & $25(44.6 \%)$ & 38.5 & \multirow[t]{2}{*}{0.04} & 1.00 & 1.00 & 1.00 & \\
\hline $\mathrm{AC}+\mathrm{CC}$ & 96 & $59(61.5 \%)$ & 17.9 & & $1.62(1.01-2.59)$ & 0.04 & $1.96(1.18-3.25)$ & 0.01 \\
\hline
\end{tabular}

(a) Median Survival Time at percentile 40th; (b) Adjusted for age, sex, Lauren, tumor size, depth of invasion and lymph node metastasis; (c) comparing survival distribution for three genotypes; (d) comparing survival distributions for carriers of minor allele and homozygous for common allele.

Hazard Ratio estimates according to clinicopathological features. Finally, the association of the selected polymorphisms with OS was assessed separately depending on the clinicopathological features of patients. For the three SNPs, HR was higher among patients with lymph node metastases $(\mathrm{N} 1, \mathrm{~N} 2$ or N3, $\mathrm{n}=111)$ compared to HR for all GC patients (rs2526614 Hausman test $p=0.0026$, rs6953766 Hausman test $p<0.0001$, rs431073 Hausman test $p=0.0391$ ) (Table IV). There were also differences in HR when comparing all GC patients versus patients with diffuse-type (rs6953766) or with tumor size $>5 \mathrm{~cm}$ (rs431073).

\section{Discussion}

EMT program has been linked to metastatic dissemination. Canonical effectors of this program include E-cadherin, Snail, Zeb, and Twist. Levels of those EMT markers have been associated with cancer survival. Nevertheless, little is known regarding the role of SNPs in genes encoding EMT markers in cancer prognosis, particularly with GC survival. In the present investigation, two SNPs in TWIST1 (rs2526614 and rs6953766), and rs431073 in ZEB1 were associated with OS in GC patients.

TWIST1 is located on chromosome 7p21.1 and encodes Twist Basic Helix-Loop-Helix Transcription Factor 1. This protein was reported to be associated with migration and invasion of GC cells (21). It is frequently expressed in tumor GC cells and also in stromal cancer-associated fibroblasts,
Table IV. Hazard Ratios of rs2526614 (TWIST1), rs6953766 (TWIST1) and rs431073 (ZEB1) according to patient's characteristics.

\begin{tabular}{lccc}
\hline Strata & $\begin{array}{c}\text { HR }(95 \% \mathrm{CI}) \\
\text { (a) }\end{array}$ & $p$-Value & $\begin{array}{c}\text { Hausman } \\
p \text {-Value }\end{array}$ \\
\hline rs2526614 (CA+AA versus CC) & & & \\
$\quad$ All patients & $0.73(0.47-1.14)$ & 0.172 & \\
$\quad$ N1, N2 or N3 & $0.57(0.36-0.92)$ & 0.022 & 0.003 \\
rs6953766 (GG versus TT) & & & \\
$\quad$ All patients & $2.15(1.13-4.10)$ & 0.020 & \\
$\quad$ Diffuse-type & $2.82(1.13-7.03)$ & 0.026 & $0.044(\mathrm{~b})$ \\
N1, N2 or N3 & $3.30(1.67-6.52)$ & 0.001 & 0.001 \\
rs431073 (AC+CC versus AA) & & & \\
$\quad$ All patients & $1.64(1.03-2.63)$ & 0.038 & \\
$\quad$ Tumor size $>5 \mathrm{~cm}$ & $2.53(1.46-4.39)$ & 0.001 & 0.009 \\
N1, N2 or N3 & $1.99(1.21-3.28)$ & 0.007 & 0.039 \\
\hline
\end{tabular}

(a) HR $(95 \% \mathrm{CI})$ Hazard Ratio (95\% Confidence Interval) adjusted for age and gender; (b) Hausman $p$-value comparing each stratum versus all patients.

but its expression was absent in gastric dysplasia, metaplasia, gastritis or normal gastric mucosa $(22,23)$. In addition, tumors expressing TWIST1 are more frequent among patients with lymph node metastases compared to N0 patients (22, 23). It is in agreement with our observation that both rs2526614 and rs6953766 have worse survival among lymph 
node-positive patients (Table IV). TWISTI expression in tumor cells has been associated with poor OS (22) and progression free survival according to data from The Cancer Genome Atlas (24). Interestingly, the expression is more frequent in diffuse-type tumors compared to intestinal tumors (23), and TWIST1 levels in stromal fibroblasts were associated with poor prognosis in the diffuse-type but not in the intestinal type (22). This fact could explain why the HR for rs6953766 was higher among patients with diffuse GC $(\mathrm{HR}=2.82,95 \% \mathrm{CI}=1.13-7.03, p=0.026)$ than intestinal $\mathrm{GC}$ $(\mathrm{HR}=1.17,95 \% \mathrm{CI}=0.40-3.40, p=0.772)$ (Table IV).

The associated polymorphisms in TWIST1 were rs 2526614 and rs6953766. rs2526614 is located at the last intron and rs6953766 on the second intron. After a search in PubMed and NHGRI-EBI GWAS catalog, neither of them nor the proxy SNPs $\left(r^{2}>0.8\right.$ in 1000 Genomes AMR population: rs2717327, rs55958613, rs2390045 and rs2390046) have been associated with any phenotype. TWIST1 rs2526614 is 300bp from its proxy variant rs $2717327\left(\mathrm{r}^{2}=1\right.$ in AMR population) and is not an expression quantitative trait loci (eQTL) according to the Genotype-Tissue Expression (GTEx) project (www.gtexportal.org). They are both located in enhancer (state 7 of Core 15 ChromHMM states) and DNAse hypersensitive sites as reported by HaploReg v4.1 (25) using data from Roadmap Epigenomics Consortium. Non-coding associated variants from GWAS studies are enriched in enhancer and DNAse hypersensitive sites $(26,27)$, therefore, a SNP present in these sites is probably functional. Also, rs2526614 is located in a binding site of $\mathrm{P} 300$ protein identified by ChiP-Seq (ENCyclopedia of DNA Elements ENCODE- data retrieved from RegulomeDB (28)). Taken together, the evidence above support, to some extent, a functional effect of rs2526614 on TWIST1 expression.

Concerning TWIST1 rs6953766, this variant is in LD with rs55958613, rs2390045 and rs2390046 in a region that spans $2.5 \mathrm{~kb}$ in intron 2 of TWIST1. The analysis of HaploReg v4.1 indicates that this region contains enhancer histone marks and DNAse hypersensitive sites. SNP2TFBS (29) was used to assess whether the associated variants and their proxy SNPs affect transcription factor (TF) binding sites in the Human genome. This tool revealed that rs6953766 creates a binding site for Spi-B transcription factor. It is an Ets family TF expressed exclusively in mature B cells, T-cell progenitors and plasmacytoid dendritic cells (30). Nevertheless, Jian et al. (31) reported that Spi-B is one of the twenty TFs overexpressed in GC tumors compared to normal tissues. Recently, Du et al. (30) published the results of a study of this TF in NSCLC tumors. They found that high Spi-B expression is associated with worst prognosis. Interestingly, down-regulation of Spi-B in an NSCLC cell line that expresses endogenous Spi-B and has a mesenchymal phenotype, resulted in up-regulation of Ecadherin and down-regulation of EMT TFs, including Twist1. The authors concluded that Spi-B might be essential in maintaining the mesenchymal phenotype of lung cancer cells. Therefore, it is possible that rs6953766 $\mathrm{G}$ allele is associated with poor OS in GC patients. This allele could create a binding site for Spi-B, a TF differentially overexpressed in GC cells, and contribute to the expression of TWIST1 in GC cells. Further studies experimentally evaluating this mechanism are warranted to support its association with OS.

$Z E B 1$ encodes zinc-finger E-box-binding homeobox factor 1 (ZEB1), a member of the zinc finger family of proteins. Down-regulation of this gene in MKN1 gastric cancer cells increased E-cadherin expression and reduced its capacity to proliferate, migrate, and invade (15). Jia et al. (32) found that this marker was frequently found in GC tumors compared to the adjacent normal gastric mucosa, in particular in poorly differentiated tumors and lymph node metastases positive patients. However, no differences were found according to tumor size $(15,32)$. These results are in agreement with our findings that show an association of rs431073 with poor prognosis, markedly among lymph nodepositive patients. On the other hand, it does not support the fact that the HR of this polymorphism is higher in patients with tumor $>5 \mathrm{~cm}(\mathrm{HR}=2.53,95 \% \mathrm{CI}=1.46-4.39)$ than in patients with tumor $<5 \mathrm{~cm}(\mathrm{HR}=0.84,95 \% \mathrm{CI}=0.32-2.19)$.

rs431073 is located in intron 1 of $Z E B 1$ and has no proxy SNPs. This polymorphism has not been associated with any phenotype. HaploReg v4.1 revealed that rs431073 is located in a region that does not contain enhancer histone marks and DNAse hypersensitive sites. This polymorphism lies in a 115 bp site identified by ChIP-seq to bind BRG1 in CD36 cells. BRG1 (encoded by SMARCA4) is a member of the SWI/SNF family of proteins involved in transcriptional activation or repression of selected genes by chromatin remodeling, and there is growing evidence about its role in cancer (33). Up to date, no studies have been published evaluating a possible role of BRG1 on the expression of $Z E B 1$. Further studies are needed to assess if rs431073 is a functional variant.

In conclusion, our study found three polymorphisms associated with OS in GC patients: TWIST1 rs2526614, TWIST1 rs6953766, and ZEB1 rs431073. The association was more noticeable when patients were stratified by certain clinicopathological features. Information from published studies as well as from databases allowed us to propose that these SNPs are functional variants. To the best of our knowledge, this is the first study proposing a role for those SNPs in GC prognosis. The use of TWIST1 rs2526614, TWIST1 rs6953766, and ZEB1 rs431073 as prognostic markers of GC survival warrants further investigation.

\section{Acknowledgements}

The Authors would like to acknowledge Benjamin García-Bloj, MD, $\mathrm{PhD}$ for his help in proofreading the manuscript. This work was supported by Fondo Nacional de Desarrollo Científico y Tecnológico -Chile- (FONDECYT) \#1151015. 


\section{References}

1 Ferlay J, Soerjomataram I, Dikshit R, Eser S, Mathers C, Rebelo M, Parkin DM, Forman D and Bray F: Cancer incidence and mortality worldwide: sources, methods and major patterns in GLOBOCAN 2012. Int J Cancer 136: E359-386, 2015.

2 Carioli G, La Vecchia C, Bertuccio P, Rodriguez T, Levi F, Boffetta P, Negri E and Malvezzi M: Cancer mortality predictions for 2017 in Latin America. Ann Oncol 28: 2286-2297, 2017.

3 Kwon OK, Kim SW, Chae H-D, Ryu SW, Chung HY, Kim SW, Lee WK and Yu W: Validation of the 7th AJCC/UICC staging system for gastric cancer and a proposal for a new TNM system based on a prognostic score: a retrospective multicenter study. Ann Surg Treat Res 91: 295-302, 2016.

4 Heise K, Bertran E, Andia ME and Ferreccio C: Incidence and survival of stomach cancer in a high-risk population of Chile. World J Gastroenterol 15: 1854-1862, 2009.

5 Compton CC, Byrd DR, Garcia-Aguilar J, Kurtzman SH, Olawaiye A and Washington MK (eds.): AJCC Cancer Staging Atlas. New York, NY, Springer New York, 2012.

6 Seeruttun SR, Yuan S, Qiu H, Huang Y, Li Y, Liang Y, Guan Y, Zhan Y, Li W, Chen Y, Sun X, Xu D and Zhou Z: A comprehensive analysis comparing the eighth AJCC gastric cancer pathological classification to the seventh, sixth, and fifth editions. Cancer Med 6: 2804-2813, 2017.

7 Mocellin S, Verdi D, Pooley KA and Nitti D: Genetic variation and gastric cancer risk: a field synopsis and meta-analysis. Gut 64: 1209-1219, 2015.

8 Sung H, Hu N, Yang HH, Giffen CA, Zhu B, Song L, Su H, Wang C, Parisi DM, Goldstein AM, Taylor PR and Hyland PL: Association of high-evidence gastric cancer susceptibility loci and somatic gene expression levels with survival. Carcinogenesis 38 : 1119-1128, 2017.

9 Jiang WG, Sanders AJ, Katoh M, Ungefroren H, Gieseler F, Prince M, Thompson SK, Zollo M, Spano D, Dhawan P, Sliva D, Subbarayan PR, Sarkar M, Honoki K, Fujii H, Georgakilas AG, Amedei A, Niccolai E, Amin A, Ashraf SS, Ye L, Helferich WG, Yang X, Boosani CS, Guha G, Ciriolo MR, Aquilano K, Chen S, Azmi AS, Keith WN, Bilsland A, Bhakta D, Halicka D, Nowsheen S, Pantano F and Santini D: Tissue invasion and metastasis: Molecular, biological and clinical perspectives. Seminars in Cancer Biology 35: S244-S275, 2015.

10 Ye X and Weinberg RA: Epithelial-mesenchymal plasticity: A central regulator of cancer progression. Trends Cell Biol 25: 675-686, 2015.

11 Nieto MA, Huang RYJ, Jackson RA and Thiery JP: EMT: 2016. Cell 166: 21-45, 2016.

12 Huang L, Wu R-L and Xu A-M: Epithelial-mesenchymal transition in gastric cancer. Am J Transl Res 7: 2141-2158, 2015.

$13 \mathrm{Ru}$ GQ, Wang HJ, Xu WJ and Zhao ZS: Up-regulation of Twist in gastric carcinoma associated with tumor invasion and poor prognosis. Pathol Oncol Res 17: 341-347, 2011.

14 Chen X, Li J, Hu L, Yang W, Lu L, Jin H, Wei Z, Yang JY, Arabnia HR, Liu JS, Yang MQ and Deng Y: The clinical significance of snail protein expression in gastric cancer: a metaanalysis. Hum Genomics 10: 22, 2016.

15 Murai T, Yamada S, Fuchs BC, Fujii T, Nakayama G, Sugimoto H, Koike M, Fujiwara M, Tanabe KK and Kodera Y: Epithelialto-mesenchymal transition predicts prognosis in clinical gastric cancer. J Surg Oncol 109: 684-689, 2014.

16 Chomczynski P and Sacchi N: The single-step method of RNA isolation by acid guanidinium thiocyanate-phenol-chloroform extraction: twenty-something years on. Nat Protoc 1: 581-585, 2006.

17 Anderson CA, Pettersson FH, Clarke GM, Cardon LR, Morris $\mathrm{AP}$ and Zondervan KT: Data quality control in genetic casecontrol association studies. Nat Protoc 5: 1564-1573, 2010.

18 Grambsch PM and Therneau TM: Proportional hazards tests and diagnostics based on weighted residuals. Biometrika 81: 515$526,1994$.

19 Hausman JA: Specification tests in econometrics. Econometrica 46: 1251-1271, 1978.

20 Schemper M and Smith TL: A note on quantifying follow-up in studies of failure time. Control Clin Trials 17: 343-346, 1996.

21 Feng M, Wang K, Song H, Yu H, Qin Y, Shi Q and Geng J: Metastasis-induction and apoptosis-protection by TWIST in gastric cancer cells. Clin Exp Metastasis 26: 1013-1023, 2009.

22 Sung CO, Lee KW, Han S and Kim SH: Twist1 is up-regulated in gastric cancer-associated fibroblasts with poor clinical outcomes. Am J Pathol 179: 1827-1838, 2011.

23 Yan Qi Z, Xue Yan G, Shuang H, Yu C, Fu Lin G, Fei Hu B, Shi-Ren S, Xu Feng W, Jie D and Dai Ming F: Expression and significance of TWIST basic helix-loop-helix protein overexpression in gastric cancer. Pathology 39: 470-475, 2007.

$24 \mathrm{Han} \mathrm{M}$ and Xu W: EMP3 is induced by TWIST1/2 and regulates epithelial-to-mesenchymal transition of gastric cancer cells. Tumor Biology 39: 101042831771840, 2017.

25 Ward LD and Kellis M: HaploReg: a resource for exploring chromatin states, conservation, and regulatory motif alterations within sets of genetically linked variants. Nucleic Acids Res 40: D930-934, 2012.

26 Ernst J, Kheradpour P, Mikkelsen TS, Shoresh N, Ward LD, Epstein CB, Zhang X, Wang L, Issner R, Coyne M, Ku M, Durham T, Kellis $\mathrm{M}$ and Bernstein BE: Mapping and analysis of chromatin state dynamics in nine human cell types. Nature 473: 43-49, 2011.

27 Schaub MA, Boyle AP, Kundaje A, Batzoglou S and Snyder M: Linking disease associations with regulatory information in the human genome. Genome Res 22: 1748-1759, 2012.

28 Boyle AP, Hong EL, Hariharan M, Cheng Y, Schaub MA, Kasowski M, Karczewski KJ, Park J, Hitz BC, Weng S, Cherry JM and Snyder M: Annotation of functional variation in personal genomes using RegulomeDB. Genome Res 22: 1790-1797, 2012.

29 Kumar S, Ambrosini G and Bucher P: SNP2TFBS - a database of regulatory SNPs affecting predicted transcription factor binding site affinity. Nucleic Acids Res 45: D139-D144, 2017.

30 Du W, Xu X, Niu Q, Zhang X, Wei Y, Wang Z, Zhang W, Yan J, Ru Y, Fu Z, Li X, Jiang Y, Ma Z, Zhang Z, Yao Z and Liu Z: Spi-B-mediated silencing of claudin-2 promotes early dissemination of lung cancer cells from primary tumors. Cancer Res 77: 4809-4822, 2017.

31 Jiang HB, Yang TJ, Lu P and Ma YJ: Gene expression profiling of gastric cancer. Eur Rev Med Pharmacol Sci 18: 2109-2115, 2014.

32 Jia B, Liu H, Kong Q and Li B: Overexpression of ZEB1 associated with metastasis and invasion in patients with gastric carcinoma. Mol Cell Biochem 366: 223-229, 2012.

33 Wu Q, Lian JB, Stein JL, Stein GS, Nickerson JA and Imbalzano AN: The BRG1 ATPase of human SWI/SNF chromatin remodeling enzymes as a driver of cancer. Epigenomics 9: 919-931, 2017.

Received April 24, 2018

Revised May 22, 2018

Accepted May 23, 2018 